\title{
An Online Study of Suicidality and its Determinants among Nigerian Young Adults Resident in China During the COVID-19 Pandemic
}

\author{
Opakunle T. ${ }^{1}$, Aloba O. ${ }^{2}$, Adebisi M. ${ }^{3}$
}

\begin{abstract}
Objective: The outbreak of coronavirus pandemic has affected millions of people globally. This has subsequently triggered diverse mental health challenges. This study examined the prevalence as well as the determinants of suicidality among Nigerian young adults resident in China.

Method: This is an online-based cross-sectional survey involving 364 Nigerian young adults in China. Sociodemographic and suicidality related variables were collected. The respondents also completed the 7-item Generalized Anxiety Disorder Questionnaire and the 5-item Brief Symptom Rating Scales.
\end{abstract}

Results: The prevalence rates of suicidal ideation and suicide attempt as a result of the pandemic were $8.2 \%$ and $2.5 \%$ respectively. A total of $188(51.6 \%)$ respondents had anxiety. Suicidality was associated with anxiety and psychological distress.

Conclusion: Suicidality is relatively common among the Nigerian young adult population in China during the COVID-19 pandemic. Mental health interventions in terms of screening for suicidality and its determinants may need to be developed for Nigerian young adults in China during this pandemic.

Keywords: Suicidality, Suicidal ideation, Suicide attempt, Covid-19 pandemic, Nigerian adults

\author{
*Corresponding Author \\ Dr. Tolulope Opakunle \\ https://orcid.org/0000-0003-0785-1476 \\ E-mail: toluopakunle@gmail.com
}

${ }^{1}$ Department of Mental Health, State Specialist Hospital, Osogbo, Osun State, Nigeria

${ }^{2}$ Department of Mental Health, Obafemi Awolowo University, Ile-Ife, Osun State, Nigeria

${ }^{3}$ Zhenjiang First People's Hospital, affiliated to Jiangsu University, People's Republic of China. 


\title{
ORIGINAL ARTICLE
}

\section{Une étude en ligne sur la suicidalité et ses déterminants chez les jeunes adultes nigérians résidant en Chine pendant la pandémie COVID-19}

\author{
Opakunle T. ${ }^{1}$, Aloba O. ${ }^{2}$, Adebisi M. ${ }^{3}$
}

\begin{abstract}
Objective: The outbreak of coronavirus pandemic has affected millions of people globally. This has subsequently triggered diverse mental health challenges. This study examined the prevalence as well as the determinants of suicidality among Nigerian young adults resident in China.

Method: This is an online-based cross-sectional survey involving 364 Nigerian young adults in China. Sociodemographic and suicidality related variables were collected. The respondents also completed the 7-item Generalized Anxiety Disorder Questionnaire and the 5-item Brief Symptom Rating Scales.
\end{abstract}

Results: The prevalence rates of suicidal ideation and suicide attempt as a result of the pandemic were $8.2 \%$ and $2.5 \%$ respectively. A total of $188(51.6 \%)$ respondents had anxiety. Suicidality was associated with anxiety and psychological distress.

Conclusion: Suicidality is relatively common among the Nigerian young adult population in China during the COVID-19 pandemic. Mental health interventions in terms of screening for suicidality and its determinants may need to be developed for Nigerian young adults in China during this pandemic.

Keywords: Suicidality, Suicidal ideation, Suicide attempt, Covid-19 pandemic, Nigerian adults

\author{
*Corresponding Author \\ Dr. Tolulope Opakunle \\ https://orcid.org/0000-0003-0785-1476 \\ E-mail: toluopakunle@gmail.com
}

${ }^{1}$ Department of Mental Health, State Specialist Hospital, Osogbo, Osun State, Nigeria

${ }^{2}$ Department of Mental Health, Obafemi Awolowo University, Ile-Ife, Osun State, Nigeria

${ }^{3}$ Zhenjiang First People's Hospital, affiliated to Jiangsu University, People's Republic of China. 


\section{INTRODUCTION}

Towards the end of 2019, a novel coronavirus (2019nCov) was identified as the prime cause of a lifethreatening pneumonia which was first reported in Wuhan, Hubei province, People's Republic of China (1). A short while later, the World Health Organization (WHO) declared the coronavirus disease - 2019 (Covid-19) as a public health emergency disease of international concern because of its rapid spread to other continents of the world within a short period of time $(1,2)$. Its clinical features include fever, dry cough, myalgia, sorethroat, anosmia, headache, abdominal pain, and diarrhea (2-5). The Covid-19 has precipitated significant mortalities globally. As at August 2020, more than 765,000 deaths have been reported worldwide. Due to the high infectivity and dangers associated with Covid-19, several countries of the world have put in place isolation and lockdown measures to combat its spread. However, these measures have mental health and socioeconomic consequences $(6,7)$. Covid-19 has not only caused severe economic problems on the population, but it has also created a panic mode in the general population (8). This has led to psychological distress and anxiety-related suicidal behaviors within many communities $(8,9)$. Therefore, suicide-related mortalities as a result of Covid-19 are not uncommon in the general population (8). They have been reported across several countries $(1,10-12)$.

In China, even though there are over 84,000 confirmed cases of Covid-19 including more than 4,600 mortalities as at August 2020, no study has investigated the suicidality related variables and their determinants among Nigerian young adults residing in China. Consequently, our study aims to determine the prevalence of suicidal behaviors (specifically suicidal ideation and attempts) during the Covid-19 pandemic period, and to examine the relationship between suicidality and its determinants which include anxiety and psychological distress among this population in China. The findings in this study may help to identify the variables that can constitute a baseline for the development of psychological interventions to mitigate severity of suicidality among the Nigerian young adults in China during this pandemic.

\section{MATERIALS AND METHODS}

\section{Study design and Participants}

In order to prevent further spread of coronavirus infection through contacts, we utilized an onlinebased cross-sectional survey. This was broadcast via the social media to our target population.

\section{Ethical approval}

Approval for the study protocol was granted by the Institutional Review Board of Zhenjiang First People's Hospital, affiliated to Jiangsu University, China.

\section{Procedure}

Participants were sent an online survey link to anonymously complete. eSurvey Creator was used to collect data from $28^{\text {th }}$ of April to $26^{\text {th }}$ May 2020. The criteria for eligibility included: 1) Nigerian adults aged between 18 and 40 years resident in China since December, 2019 till the time of the survey, 2) No existing psychiatric disorder, and 3) No underlying chronic medical condition.

\section{Studymeasures}

\section{Sociodemographic questionnaire}

This consisted of variables such as the age, nationality, gender, and duration of stay in China.

\section{A study specific suicidality questionnaire}

Suicide related behaviours were assessed with two close-ended questions (Yes or No). The first question was to determine if the respondent has been having suicidal ideations and the second question enquired if the respondent has attempted suicide in the context of the covid-19 pandemic.

\section{Generalized Anxiety Disorder - 7 (GAD-7)}

The GAD-7 was used to assess the symptoms of anxiety disorder as a result of the pandemic. A unit of $0,1,2$, and 3 , was allocated to the respondents' answers to each questions ('not at all- 0 ', 'several days1 ', 'more than half the days-2', and 'nearly every day$\left.3^{\prime}\right)$. Total scores of 5, 10, and 15 were respectively the cut-off points for mild, moderate, and severe anxiety (13). It has been used and validated in the Chinese population and found to have good reliability (14, $15)$. It has also been used among the Nigerian population(16).

\section{Brief Symptom Rating Scale-5 (BSRS-5)}

The BSRS-5 contained five subjectively completed 
items that quantified psychological symptoms as a result of the pandemic (17). It was derived from the original 50-item scale which measures five domains namely anxiety, depression, hostility, interpersonal sensitivity, and other psychological symptoms. The score for each item ranges from 0 to 4 ( 0 , not at all; 1 , a little bit; 2 , moderately; 3 , quite a bit; and 4 , extremely)(17).

\section{Data analysis}

All statistical analyses were performed with the Statistical Product for Service Solutions (SPSS), 21st version. Descriptive statistics such as the mean (standard deviation) and frequency (percentages) were utilized in depicting the sociodemographic variables and scores on the study measures. The dependent variables were suicidal ideation and suicide attempt. Binary logistic regression analyses were conducted to identify the variables that were predictive of dependent variables. All statistical analyses were 2-tailed, and the level of statistical significance was set at a $p$ value less than 0.05 .

\section{RESULTS}

A total of 382 Nigerians across China participated in this survey. However, 15 questionnaires were incompletely filled and three non-Nigerians that responded to the survey were excluded. Hence, only 364 survey responses were available for data analysis.

Table 1 shows that the mean age of the respondents was 29.92 (SD 5.60) years. Males constituted $53.8 \%$ of the total sample. Those who indicated suicidal ideation and had attempted suicide during the period of the pandemic were $8.2 \%$ and $2.5 \%$ respectively. A total of $188(51.6 \%)$ had anxiety symptoms (mild, moderate, and severe anxiety symptoms were $19.2 \%, 16.5 \%$ and $15.9 \%$ respectively).

Table 1: Sociodemographic characteristics of the respondents $(n=364)$

\begin{tabular}{lc}
\hline Variable & N (\%) Mean (SD)[Range] \\
\hline Age & $29.92(5.60)[19-39]$ \\
Gender & \\
\multicolumn{1}{l}{ Male } & $196(53.8)$ \\
Female & $168(46.2)$ \\
Suicidal Ideation & $30(8.2)$ \\
Suicidal Attempt & $9(2.5)$ \\
GAD & $7.08(6.49)[0-22]$ \\
BSRS & $6.67(4.59)[0-19]$ \\
Generalized Anxiety Disorder & $176(48.4)$ \\
$\quad$ No Anxiety & $70(19.2)$ \\
$\quad$ Mild Anxiety & $60(16.5)$ \\
$\quad$ Soderate Anxiety & $58(15.9)$ \\
\hline
\end{tabular}

GAD - Generalized Anxiety Disorder; BSRS - Brief Symptom Rating Scale

Table 2: Gender differences in anxiety among the respondents

\begin{tabular}{llllll}
\hline Variable n (\%) & Male & Female & & & \\
& N (\%) & N (\%) & $\chi^{2}$ & df & $\boldsymbol{p}$ \\
\hline No Anxiety - 176(48.4) & $103(52.6)$ & $73(43.5)$ & 2.999 & 1 & 0.083 \\
Anxiety - 188(51.6) & $93(47.4)$ & $95(56.5)$ & & & \\
\hline
\end{tabular}

Table 3: Logistic regression showing variables that are significantly associated with suicidal ideation among the respondents

\begin{tabular}{lllllllc}
\hline Variable & B & SE & Wald & df & $\boldsymbol{P}$ & OR & 95\% CI \\
\hline GAD & 0.462 & 0.074 & 38.945 & 1 & $<0.001$ & 1.587 & $1.373-1.835$ \\
BSRS & -0.176 & 0.066 & 7.004 & 1 & 0.008 & 0.839 & $0.736-0.955$ \\
Age & -0.015 & 0.048 & 0.099 & 1 & 0.753 & 0.985 & $0.897-1.082$ \\
\hline
\end{tabular}

GAD - Generalized Anxiety Disorder; BSRS - Brief Symptom Rating Scale; B - Beta; SE - Standard Error; OR - Odd Ratio; CI- Confidence Interval 
Table 4: Logistic regression showing variables that are significantly associated with suicidal attempt among the respondents

\begin{tabular}{llllllll}
\hline Variable & B & SE & Wald & df & $\boldsymbol{P}$ & OR & 95\% CI \\
\hline GAD & 0.035 & 0.068 & 0.262 & 1 & 0.609 & 1.036 & $0.906-1.184$ \\
BSRS & 0.215 & 0.095 & 5.164 & 1 & 0.023 & 1.240 & $1.030-1.493$ \\
Age & -0.013 & 0.067 & 0.036 & 1 & 0.849 & 0.987 & $0.865-1.126$ \\
\hline
\end{tabular}

GAD - Generalized Anxiety Disorder; BSRS - Brief Symptom Rating Scale; B - Beta;

SE - Standard Error; OR - Odd Ratio; CI- Confidence Interval

Table 2 showed that there was no gender difference in relation to anxiety among the respondents $\left(\chi^{2}=2.999, P=0.083\right)$.

According to Table 3, those respondents who had indicated suicidal ideation had a higher odd (approximately 1.6) of reporting more anxiety symptoms and a higher odd (approximately 0.8) of reporting more psychological distress. Table 4 showed that the respondents who indicated that they had attempted suicide had a higher odd (approximately 1.2) of experiencing greater levels of psychological distress.

\section{DISCUSSION}

This study examined the components of suicidality and its determinants among Nigerian young adults that were resident in China during covid-19 pandemic. The prevalence rates of suicidal ideation and suicide attempt were $8.2 \%$ and $2.5 \%$ respectively. An extensive electronic literature review revealed that no study has examined suicidality and its determinants among Nigerian young adults in China. In addition, suicidal behavior data is scarce among young adults in Asian cultures (18). Nevertheless, the results of this study were similar to the prevalence rates of $8.4 \%$ and $2.5 \%$ reported for suicidal ideation and suicide attempt among adults across three Asian countries (18). However, our findings were lower than the rates of $17.5 \%$ and $4.9 \%$ for suicidal ideation and suicide attempt reported among the general adult population in the United States of America during this pandemic (19).

Furthermore, more than half of the respondents in this study reported anxiety symptoms. This is not unusual as anxiety symptoms have been reported to increase during infectious disease outbreaks (20). It has been suggested that the increase in the symptoms of anxiety may be attributable to the devastating and dramatic covid-19 pandemic news headlines, erroneous or contradictory reports about the infectious agent transmission and symptoms, fear of the unknown and economic issues precipitated within the population as a result of the pandemic (1). The prevalence of anxiety symptoms $(51.6 \%)$ in our study is higher than the prevalence of $34.0 \%$ reported earlier in the general population in China, and $44.7 \%$ reported among the Chinese medical workers (20, 21). This contrast could be due to cultural differences in the expression of anxiety symptoms (22). One other plausible reason may be because the data were collected at different periods of the covid-19 pandemic.

There was no statistically significant gender difference in the expression of anxiety in our study, although, anxiety symptoms are more severe among females $(23,24)$. It is plausible that there are other unexplored psychosocial factors related to the novel covid-19 pandemic that brings about the obliteration of the typically observed gender anxiety differences. It is recommended that more studies are needed regarding this observation.

Furthermore, our study showed that both anxiety and psychological distress significantly determine suicidal ideation, while psychological distress was the only determinant of suicide attempt among our respondents following binary logistic regression. This buttressed an earlier study which reported that covid-19 related experiences such as anxiety and psychological distress were associated with suicidal behaviours (19). In addition, other previous studies on large scaled infectious diseases have shown that pandemic-related anxiety are usually associated with components of suicidality (25). It has been postulated that social isolation, lockdown measures and impending economic measures are some of the identified factors that could provoke components of suicidality in individuals who are already experiencing anxiety and psychological distress in the context of the covid-19 pandemic (26).

This study is not without limitations. One is that it was cross-sectional in nature, hence, the direction 
of causality cannot be categorically established. The possibilities of both recall and reporting bias regarding the study measures cannot be eliminated since they were self-reported. Selection bias may also be a limitation since our data was collected online. Nonetheless, the strength of our study is that the findings could serve as baseline statistics for comparison during the COVID-19 pandemic with other cultural settings, in addition to providing the variables that will be the templates on which the psychosocial interventions for Nigerian young adults in China may be established.

\section{CONCLUSIONS}

This study has shown that suicidal ideation and attempt are present in a sample of Nigerian young adults living in China during the covid-19 pandemic. The study also revealed that anxiety and psychological distress were significantly associated with suicidality among them.

\section{ACKNOWLEDGEMENT}

We appreciate those who favorably responded to the online survey.

\section{FINANCIAL DISCLOSURE}

None.

\section{CONFLICT OF INTEREST}

There was no conflict of interest.

\section{REFERENCES}

1. Shigemura J, Ursano RJ, Morganstein JC, Kurosawa M, Benedek DM. Public responses to the novel 2019 coronavirus (2019-nCoV) in Japan: mental health consequences and target populations. Psychiat Clin Neuros. 2020;74(4):281. Doi: 10.1111/pcn.12988.

2. Sohrabi C, Alsafi Z, O'Neill N, Khan M, Kerwan A, Al-Jabir A, et al. World Health Organization declares global emergency: A review of the 2019 novel coronavirus (COVID-19). Int. J. Surg. 2020. 76 (4): 71-76. Doi: 10.1016/j.ijsu.2020.02.034.

3. Lai CC, Shih TP, Ko WC, Tang HJ, Hsueh PR. Severe acute respiratory syndrome coronavirus 2 (SARSCoV-2) and corona virus disease-2019 (COVID-19): the epidemic and the challenges. Int. J. Antimicrob. Agents. 2020:105924. Doi: 10.1016/j.ijantimicag. 2020.105924.
4. Del Rio C, Malani PN. COVID-19 - new insights on a rapidly changing epidemic. JAMA. 2020;323 (14):1339-40. Doi:10.1001/jama.2020.3072.

5. Cascella M, Rajnik M, Cuomo A, Dulebohn SC, Di Napoli R. Features, evaluation and treatment coronavirus (COVID-19). Statpearls [internet]: StatPearls Publishing; 2020.

6. Pfefferbaum B, North CS. Mental health and the Covid-19 pandemic. N. Engl. J. Med. 2020. Doi: 10.1056/NEJMp2008017.

7. Sher L. An infectious disease pandemic and increased suicide risk. Braz J Psychiatry. 2020. 42(3):239-240. Doi: 10.1590/1516-4446-2020-0989.

8. Sahoo S, Bharadwaj S, Parveen S, Singh AP, Tandup C, Mehra A, et al. Self-harm and COVID-19 Pandemic: An emerging concern-A report of 2 cases from India. Asian J. Psychiatr. 2020. Doi: 10.1016/j.ajp.2020.102104.

9. Sher L. COVID-19, anxiety, sleep disturbances and suicide. Sleep Med. 2020. 70(124). Doi: 10.1016/j.sleep.2020.04.019.

10. Goyal K, Chauhan P, Chhikara K, Gupta P, Singh MP. Fear of COVID 2019: First suicidal case in India! Asian J. Psychiatr. 2020;49:101989. Doi: 10.1016/j.ajp.2020.101989.

11. Mamun MA, Griffiths MD. First COVID-19 suicide case in Bangladesh due to fear of COVID-19 and xenophobia: Possible suicide prevention strategies. Asian J. Psychiatr. 2020;51:102073. Doi: 10.1016/j.ajp.2020.102073.

12. Reger MA, Stanley IH, Joiner TE. Suicide Mortality and Coronavirus Disease 2019-A Perfect Storm? JAMA Psychiatry. 2020. Doi:10.1001/jama psychiatry.2020.1060.

13. Spitzer RL, Kroenke K, Williams JB, Löwe B. A brief measure for assessing generalized anxiety disorder: the GAD-7. Arch. Intern. Med. 2006;166(10):1092-7. Doi:10.1001/archinte.166.10.1092.

14. Tong X, An D, McGonigal A, Park S-P, Zhou D. Validation of the Generalized Anxiety Disorder-7 (GAD-7) among Chinese people with epilepsy. Epilepsy Res. 120, 31-36. Doi: 10.1016/j.eplepsyres. 2015.11.019.

15. Wang Y, Chen R, Zhang L. Reliability and validity of generalized anxiety scale-7 in inpatients in Chinese general hospital. J Clin Psychiatr. 2018;28:168-71. Doi: 10.3969/j.issn.1005-3220.2018.03.007.

16. Okwaraji FE, Aguwa EN, Shywobi-Eze C, Nwokpoku EN, Nduanya CU. Psychosocial impacts of communal conflicts in a sample of secondary 
school youths from two conflict communities in south east Nigeria. Psychol Health Med. 2017;22(5):58895. Doi: 10.1080/13548506.2016.1192655.

17. Lu IC, Jean M-CY, Lei S-M, Cheng H-H, Wang JD. BSRS-5 (5-item Brief Symptom Rating Scale) scores affect every aspect of quality of life measured by WHOQOL-BREF in healthy workers. Qual Life Res. 2011;20(9):1469-75. Doi: 10.1007/s11136-0119889-4.

18. Blum R, Sudhinaraset M, Emerson MR. Youth at risk: suicidal thoughts and attempts in Vietnam, China, and Taiwan. J Adolescent Health. 2012;50(3):37-44. Doi: 10.1016/j.jadohealth.2011.12.006.

19. Ammerman BA, Burke TA, Jacobucci R, McClure K. Preliminary Investigation of the Association Between COVID-19 and Suicidal Thoughts and Behaviors in the US. 2020. https://doi.org/10.31234/osf.io/68djp. Accessed 14/6/2020.

20. Huang Y, Zhao N. Generalized anxiety disorder, depressive symptoms and sleep quality during COVID-19 outbreak in China: a web-based crosssectional survey. Psychiatry Res. 2020:112954. Doi: 10.1016/j.psychres.2020.112954.

21. Liu S, Yang L, Zhang C, Xiang Y-T, Liu Z, Hu S, et al. Online mental health services in China during the
COVID-19 outbreak. Lancet Psychiat. 2020;7(4):178. Doi: 10.1016/S2215-0366(20)30077-8.

22. Dsouza DD, Quadros S, Hyderabadwala ZJ, Mamun M. Aggregated COVID-19 suicide incidences in India: Fear of COVID-19 infection is the prominent causative factor. Psychiatry Res. 2020. 290:113145. Doi: 10.1016/j.psychres.2020.113145.

23. Gao W, Ping S, Liu X. Gender differences in depression, anxiety, and stress among college students: a longitudinal study from China. J. Affect. Disord. 2020;263:292-300.

24. Guo X, Meng Z, Huang G, Fan J, Zhou W, Ling W, et al. Meta-analysis of the prevalence of anxiety disorders in mainland China from 2000 to 2015. Sci. Rep. 2016;6(1):1-15. Doi: 10.1038/srep28033.

25. Wheaton MG, Abramowitz JS, Berman NC, Fabricant LE, Olatunji BO. Psychological predictors of anxiety in response to the H1N1 (swine flu) pandemic. J. Cogn. Psychother. 2012;36(3):210-8. Doi:10.1007/ s10608-011-9353-3.

26. Thakur V, Jain A. COVID 2019-suicides: A global psychological pandemic. Brain Behav Immun. 2020. S0889-1591(20)30643-7. Doi: 10.1016/j.bbi. 2020.04.062. 